\title{
Trends in the Use of Augmented Reality, Virtual Reality, and Mixed Reality in Surgical Research: a Global Bibliometric and Visualized Analysis
}

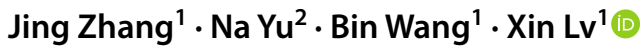

Received: 14 May 2021 / Accepted: 11 December 2021 /Published online: 24 February 2022

(c) Association of Surgeons of India 2022

\begin{abstract}
There have been many major developments in the use of augmented reality (AR), virtual reality (VR), and mixed reality (MR) technologies in the context of global surgical research, yet few reports on the trends in this field have been published to date. This study was therefore designed to explore these worldwide trends in this clinically important field. Relevant studies published from 1 January 2009 through 13 October 2020 were retrieved from the Science Citation Index-Expanded (SCI-E) tool of the Web of Science database. Bibliometric techniques were then used to analyze the resultant data, with visual bibliographic coupling, co-authorship, co-citation, co-occurrence, and publication trend analyses subsequently being conducted with GraphPad Prism 8 and with the visualization of similarities (VOS) software tool. There is no patient and public involved. In total, 6221 relevant studies were incorporated into this analysis. At a high level, clear global annual increases in the number of publications in this field were observed. The USA made the greatest contributions to this field over the studied period, with the highest H-index value, the most citations, and the greatest total link strength for analyzed publications. The country with the highest number of average citations per publication was Scotland. The Surgical Endoscopy And Other Interventional Techniques journal contributed the greatest number of publications in this field. The University of London was the institution that produced the greatest volume of research in this field. Overall, studies could be broadly classified into five clusters: Neurological Research, Surgical Techniques, Technological Products, Rehabilitative Medicine, and Clinical Therapy. The trends detected in the present analysis suggest that the number of global publications pertaining to the use of AR, VR, and MR techniques in surgical research is likely to increase in the coming years. Particular attention should be paid to emerging trends in related fields including MR, extended reality, head-mounted displays, navigation, and holographic images.
\end{abstract}

Keywords AR VR and MR $\cdot$ Surgery $\cdot$ Bibliometrics $\cdot$ Visualized analysis $\cdot$ Research trends

$\begin{array}{ll}\text { Abbreviations } \\ \text { IF } & \text { Impact factor } \\ \text { TLS } & \text { Total link strength } \\ \text { WOS } & \text { Web of Science }\end{array}$

Jing Zhang and $\mathrm{Na}$ Yu contributed equally to this work.

Xin Lv

doctor18834180608@163.com

1 Orthopedic Department, Second Hospital of Shanxi Medical University, Taiyuan 030001, China

2 Cardiology Department, Second Hospital of Shanxi Medical University, Taiyuan, China

\section{Introduction}

Virtual reality (VR) adds some substances and elements of the real world to the virtual space to make people feel the existence of the real world. Augmented reality (AR) adds a certain amount of virtual components and elements in the real space to make people feel that they have entered the virtual world. Mixed reality (MR) is the fusion of real space and virtual space, and artificial interaction in the real world and virtual world. The concept of VR first emerged in the 1960s, when Tom Furness developed technology that enabled jet pilots to access three-dimensional (3D) avionic data [1]. Many recent advances in VR and the related fields of AR and MR have been made in commercial contexts, with a range of VR systems harboring integrated haptic feedback peripherals having first been marketed to consumers in the 1980s and 1990s, although 
these platforms were expensive and suffered from relatively poor performance [2]. The limited commercial success of these VR/AR technologies led to their initial utilization primarily in academic or corporate contexts, leading to increased interest in their medical application [2]. The emergence of MR platforms has been particularly valuable in this space, as this hybrid of AR and VR technologies overcomes the respective limitations of these technologies by enabling users to interact with 3D data packets without excluding the surrounding real-world environment [3]. This hybrid MR approach thus enables the simultaneous manipulation of both real and virtual environments $[4,5]$. Sustained advances in the computing power underlying these altered reality-based technologies can subvert many healthcare-related fields, with surgery being a particularly promising context for the application of these novel tools [6]. Indeed, the combination of AR, VR, or MR techniques with robotic-assisted surgery has the potential to greatly improve surgical precision while decreasing operative durations and alleviating surgeon fatigue [3]. These technologies also offer clear value in contexts including medical education, doctorpatient communication, preoperative planning, intraoperative guidance and navigation, teleconsultation, and other surgeryrelated fields [7]. Consistent with such value, total spending on AR and VR products is forecast to exceed $\$ 215$ billion in 2021, and the global healthcare AR and VR market is forecast to reach $\$ 5.1$ billion by 2025 [2]. Many major medical and technological companies are thus seeking to develop novel approaches to combining surgical techniques and computer imaging modalities to achieve key breakthroughs, making a timely analysis of the application of VR, AR, and MR technologies in the field of surgical research of key importance. Few such studies have been published to date, highlighting the need to examine current and future trends in this research space in an effort to both understand and guide the directions of studies in this innovative and exciting field.

Peer-reviewed publications are a primary output associated with scientific research, and can be readily analyzed to gauge contributions to a given research field [8]. Bibliometric analyses and corresponding visual mapping techniques have been routinely utilized in recent years to assess scientific progress at a high level [9]. These analyses focus on the quantitative and qualitative evaluation of trends in a given research community over time based on data obtained from key online databases [10], comparing the relative contributions of different scholars, journals, institutions, and countries to a field of interest [11]. Bibliometric analyses have previously been leveraged to guide health policy and clinical guideline development, and to improve understanding of a range of conditions and technologies [12], including Middle East respiratory syndrome coronavirus (MERS-CoV) [13], exosomes [14], retinal regeneration [15], and spinal ultrasound [16]. The present study was therefore designed to assess current and future trends pertaining to the use of
AR, VR, and MR technologies in the field of surgery and to identify emerging topics in this research space that are the focus of growing scholarly investigation.

For this analysis, VR was defined as a computer-generated 3D-simulated environment in which users are able to fully immerse themselves, while AR was defined as the projection of computer-generated images onto real-world entities, and MR was defined as the AR-like projection of virtual objects onto real-world entities such that these objects were responsive and spatially aware such that users were able to interact with these $3 \mathrm{D}$ projections $[6$, 7] (Fig. 1).

\section{Materials and Methods}

\section{Data Sources}

Bibliometric analyses were conducted by using the Science Citation Index-Expanded of the Web of Science (WOS), as this is the standard database used for such analyses [17]. No ethical oversight was required for this study, as it did not involve human subjects or preclinical animal models.

\section{Search Strategy}

The WOS database was searched for relevant studies published from January 2009 to October 2020 using the following search terms: (TS = "virtual reality" OR "augmented reality" OR "mixed reality") AND (Language=English) AND (Document type = Articles OR Reviews). Key publication-related information including countries or regions of origin, institutions, funding sources, and research directions were then obtained from the WOS database. Studies were excluded from this analysis if they were unrelated to Surgery, including studies of Chemistry, Computer Theory, and Electrical Engineering.

\section{Data Collection}

Records for all studies identified through the above search strategy including title, year of publication, author names, institutional affiliations, nationality, keywords, abstracts, and publishing journals were saved in a.txt file format using the WOS and were opened in Microsoft Excel 2019 for analysis. Two co-authors (ZJ and $\mathrm{YN}$ ) independently extracted data from these studies, with any discrepancies in extracted data being resolved through discussion or by contacting relevant experts. 


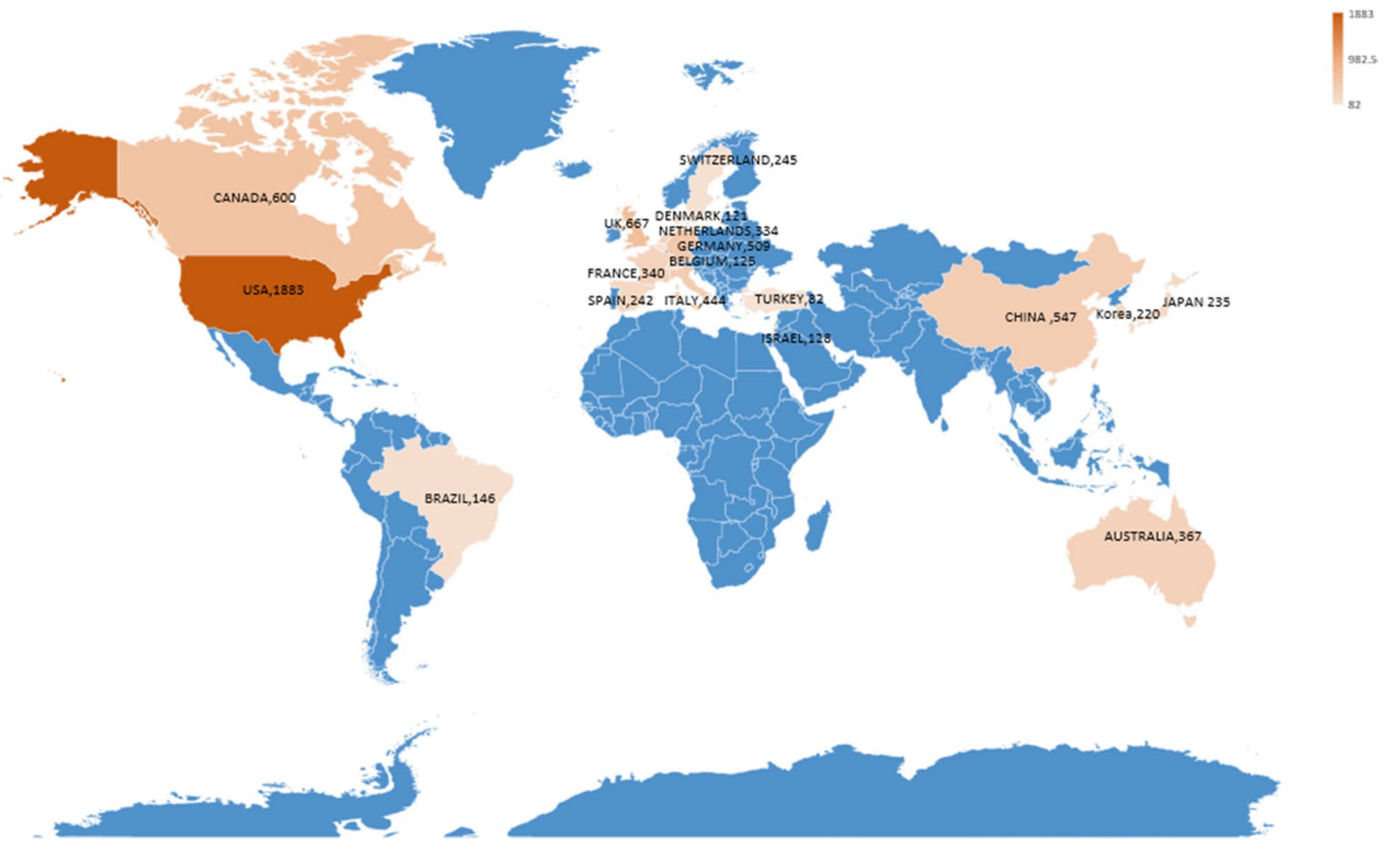

Fig. 1 A map highlighting numbers of studies pertaining to the use of these technologies in surgery that have been published in different counties, with color depth corresponding to the numbers of publications per country

\section{Bibliometric Analysis}

Bibliometric analyses leverage bibliometric theory to analyze relevant studies through statistical and mathematical approaches, thereby offering high-level insights into specific areas of scientific interest $[18,19]$. Intrinsic WOS tools can be used to assess the characteristics of studies included in bibliometric analyses. H-index values, for example, serve as key bibliometric indicators of scientific impact. $\mathrm{H}$-values used to compute the $\mathrm{H}$-index are based upon the number of times that publications from a given institution or researcher have been cited by other publications, thereby reflecting both the number of publications and the number of citations per published article [20]. The impact factor (IF) values for all journals in the present analysis were derived from the 2019 Journal Citation Reports.

\section{Visualized Analysis}

Past and predicted changes in publication volume over time were evaluated using a curve fitting model $(Y=A X-B)$. Data pertaining to time-dependent trends in key bibliometric parameters were analyzed using GraphPad Prism (GraphPad Software Inc., CA, USA) [21]. VOS viewer (Leiden University, Leiden, Netherlands) was used for visual analyses of the bibliometric data and to conduct bibliographic coupling, co-authorship, co-citation, and co-occurrence analyses.

\section{Results}

\section{Global Publication Trends}

\section{Numbers of Publications}

The number of scientific articles published in a particular field can offer key insight into current research trends in a given area. In total, we identified 6221 relevant studies in the WOS database published from 1 January 2009 to 13 October 2020 that met our search criteria, of which the majority $(3559,57.2 \%)$ were published within the last 5 years. We detected an overall upward trend in publications in this field over the past 12 years, suggesting that scholarly focus regarding the use of VR, AR, and MR in surgical contexts will continue to grow rapidly for the foreseeable future.

\section{Country-Specific Contributions}

The top 20 nations to have contributed to this field of research were next identified (Fig. 2). The greatest number of studies and reviews included in this analysis was published by 


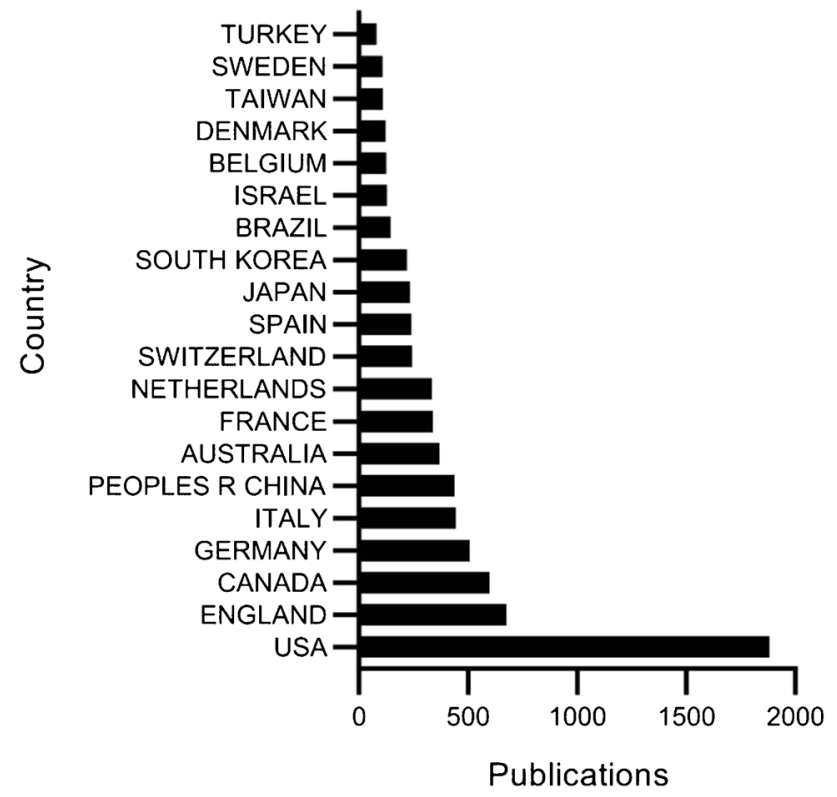

Fig. 2 The total number of publications analyzing the use of AR, VR, and MR in surgical contexts from the top most prolific nations

research groups from the USA $(1883,30.27 \%)$, followed by those from England $(677,9.82 \%)$, Canada $(600,9.64 \%)$, Germany (509, 8.18\%), and Italy 444, 7.14\%) (Figs. 3, 4, and 5).

\section{Institutional Contributions}

The top 20 institutions and organizations to have engaged in research regarding the use of AR, VR, and MR in surgery are shown in Fig. 6. Of these, the University of London has published the greatest number of studies (188), followed by the University of Toronto (44), and Imperial College London (35).

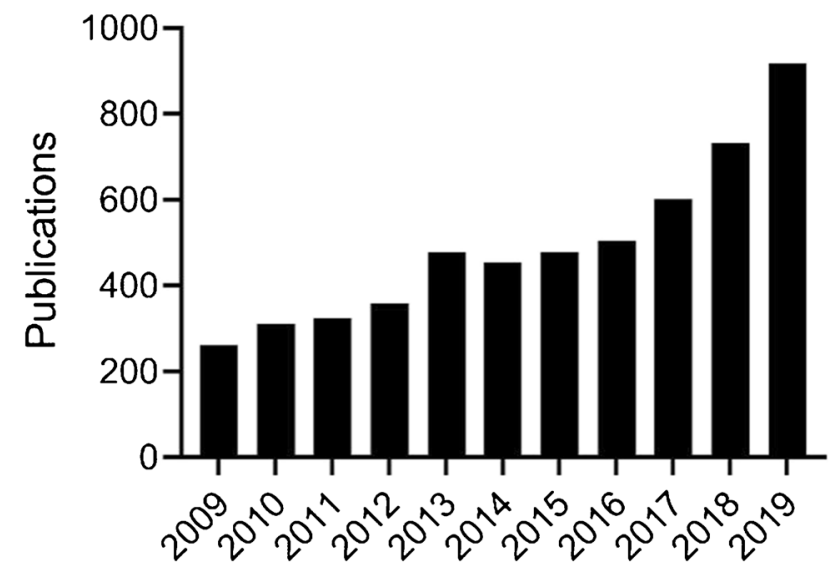

Fig. 4 Annual numbers of publications related to the use of VR, AR, and MR in surgery published over the past 11 years. Institutional, research orientation, funding source, author, and journal contributions to this field of research

\section{Contributions of Research Disciplines}

The top three research areas that explored the use of AR, VR, and MR in surgery were the Surgery, Neuroscience, and Rehabilitation fields (Fig. 7).

\section{Funding Source Contributions}

The top 4 most commonly noted funding sources for studies included in this analysis were the US Department of Health and Human Services (413), the National Institutes of Health (NIH, USA) (394), the National Natural Science Foundation of China (175), and the National Institute for Health Research (NIHR)(112), as shown in Fig. 8.
Fig. 3 Model fitting curves were used to track trends in the future publication of studies pertaining to this topic of interest
Publications

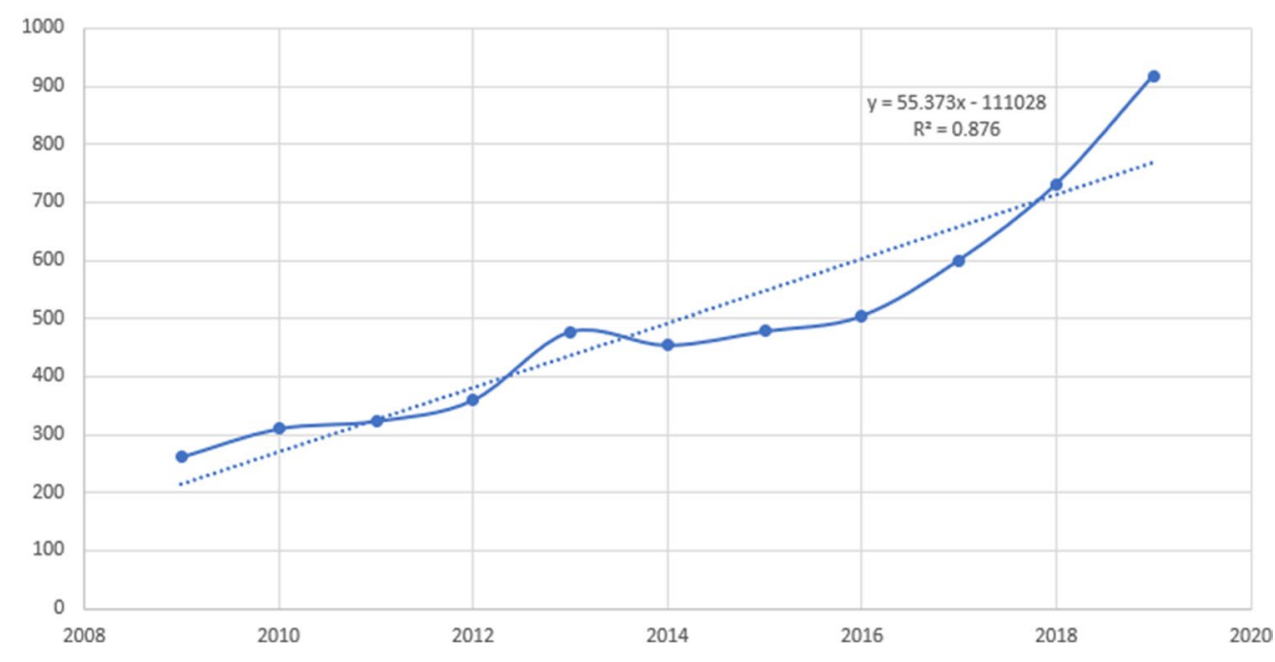


Fig. 5 Institutions publishing the most studies pertaining to the use of AR, VR, and MR in surgery ranked according to number of publications

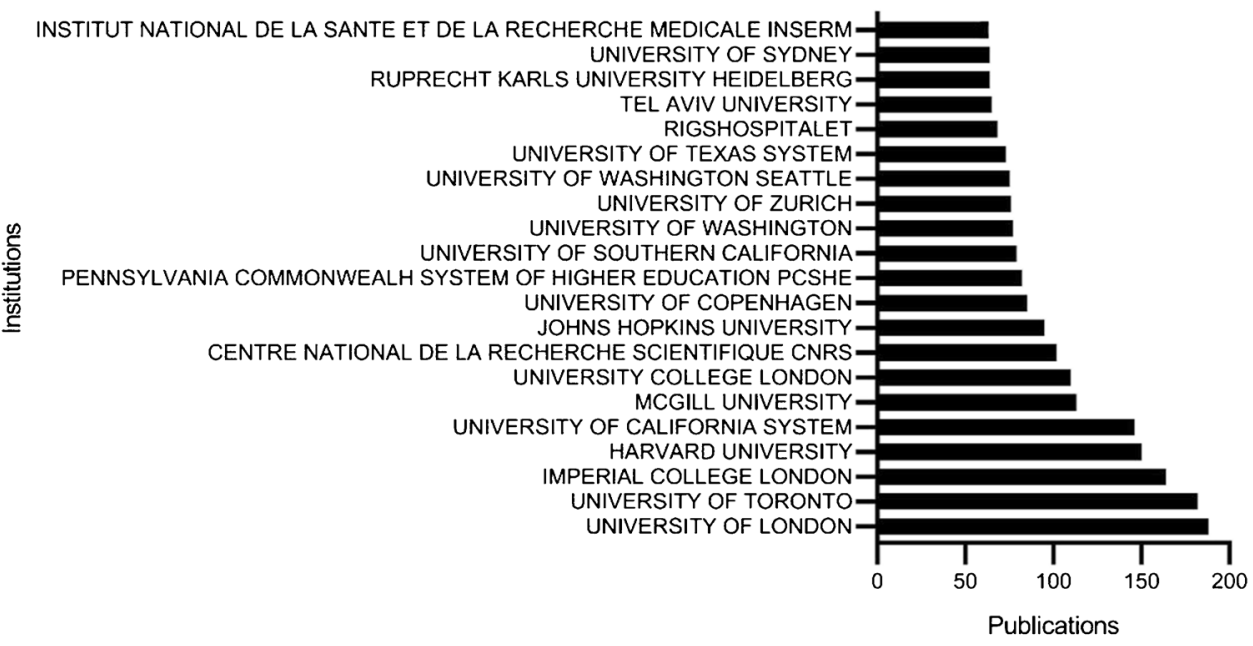

Fig. 6 Research orientations publishing the most studies pertaining to this field of interest ranked according to numbers of publicationss

Fig. 7 The most cited funding sources for studies pertaining to this field of interest ranked according to numbers of citing publications
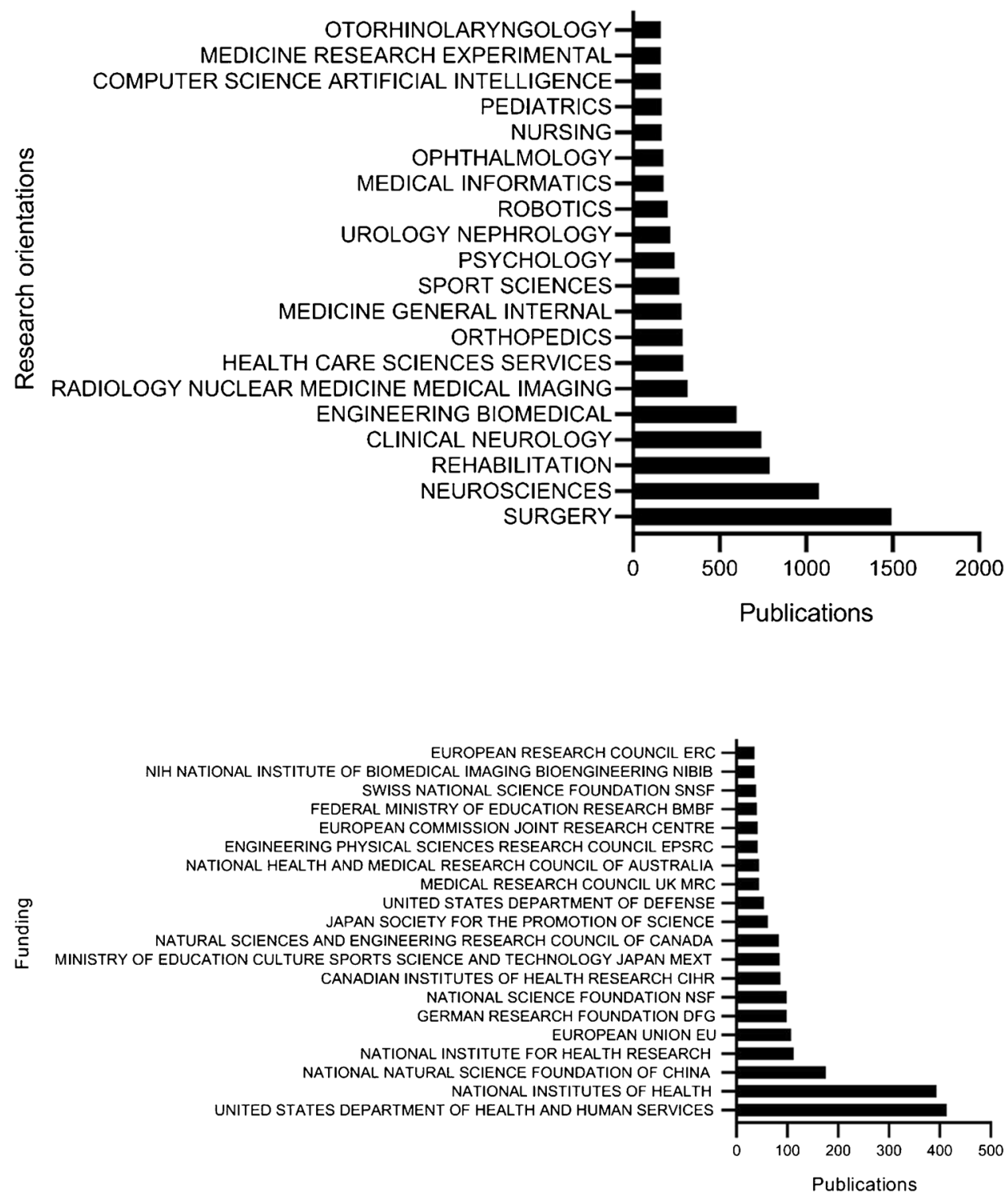


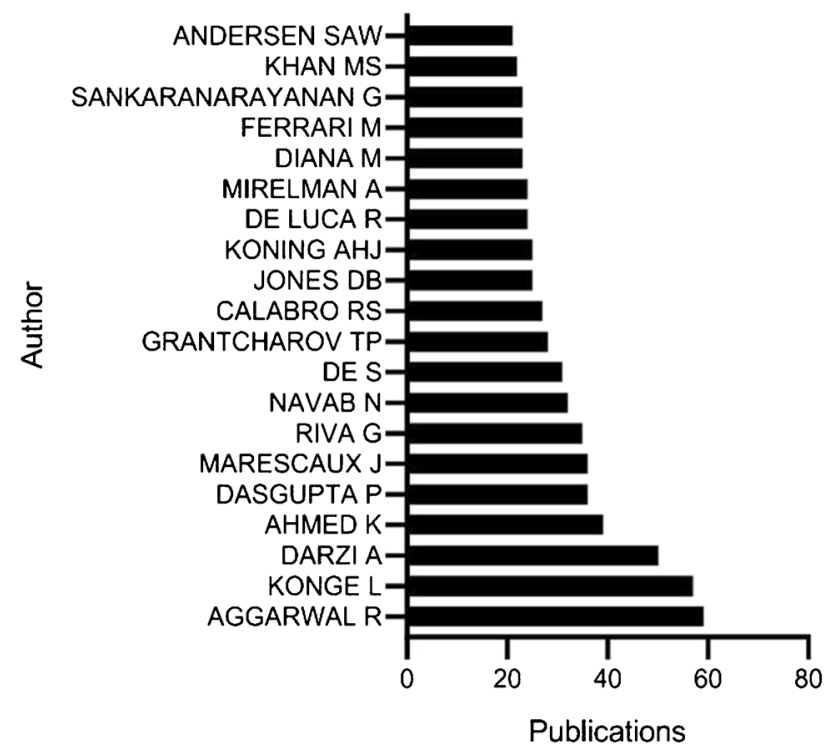

Fig. 8 The authors publishing the most studies pertaining to this field of interest ranked according to numbers of publications

\section{Contributions of Authors}

The top 3 authors in this field were Aggarwal R, Konge L, and Darzi A, who respectively published 59, 57, and 50 articles in this research space (Fig. 9).

\section{Journal Contributions}

The top 5 journals publishing the most studies pertaining to the use of AR, VR, and MR in surgery identified in the present analysis were Surgical Endoscopy And Other Interventional Techniques $(n=214)$, International Journal Of Computer Assisted Radiology And Surgery $(n=137)$, Frontiers In Human Neuroscience ( $n=133)$, IEEE Transactions On Neural Systems And Rehabilitation Engineering $(n=72)$, and the International Journal Of Medical Robotics And Computer Assisted Surgery $(n=71)$ (Fig. 10, Tables 1 and 2).

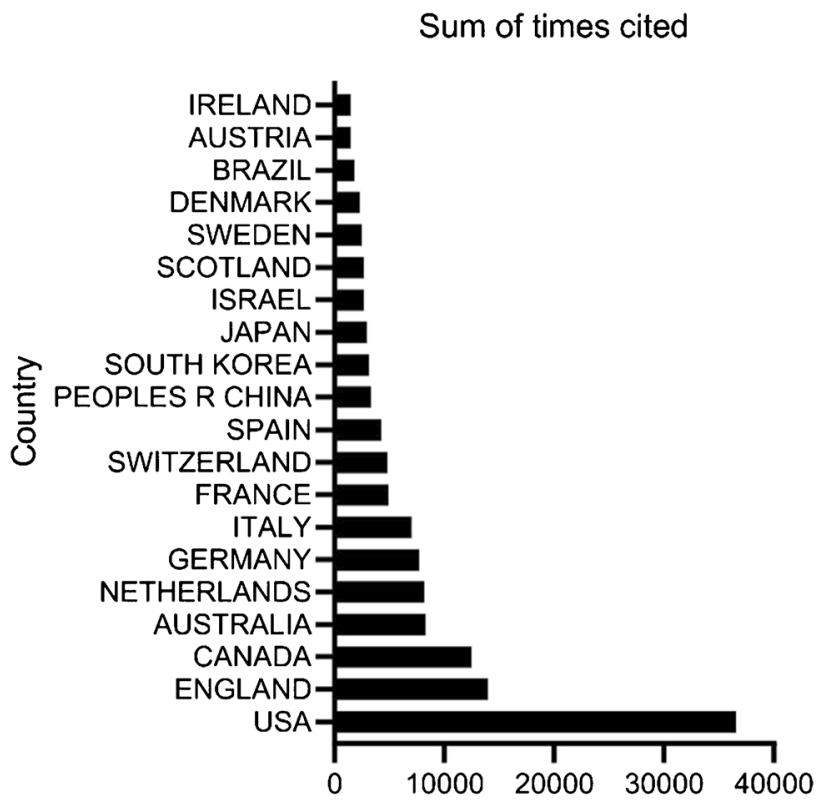

Fig. 10 Total numbers of citations for studies pertaining to AR, VR, and MR use in surgery from the indicated countries

\section{Quality of Publications from Individual Countries}

\section{Total Citation Frequency}

Studies from the USA had the greatest number of citations $(36,577)$, followed by those from England $(13,969)$, Canada (12,446), Australia (8290), and the Netherlands (8187) (Fig. 11).

\section{Average Citation Frequency}

Publications from Scotland exhibited the greatest average number of citations per study (33.08), followed by those from Austria (32.91), the Netherlands (80.74), Australia (22.59), and Sweden (22.52) (Fig. 12).
Fig. 9 Journals publishing the most studies pertaining to this field of interest ranked according to numbers of publications. Citation frequencies and $\mathrm{H}$-index values for individual nations

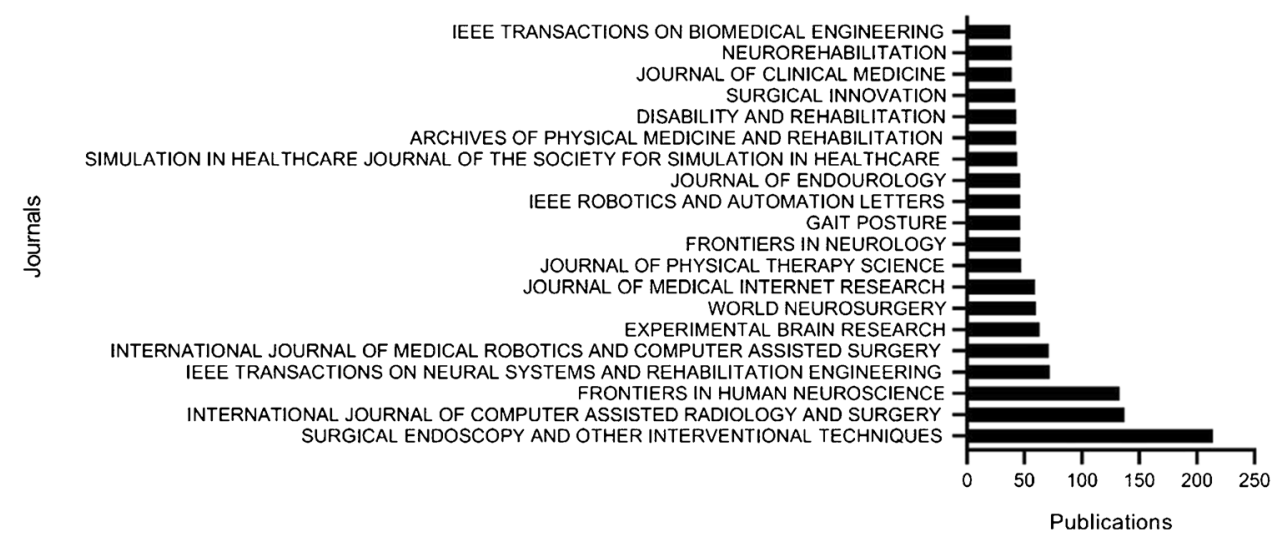


Table 1 The number of publications in the top 20 countries or regions

\begin{tabular}{lll}
\hline Country & Articles & H-index \\
\hline USA & 1883 & 81 \\
England & 677 & 55 \\
Canada & 600 & 55 \\
Germany & 509 & 38 \\
Italy & 444 & 40 \\
People's Republic of China & 437 & 27 \\
Australia & 367 & 41 \\
France & 340 & 34 \\
Netherlands & 334 & 41 \\
Switzerland & 245 & 39 \\
Spain & 242 & 36 \\
Japan & 235 & 18 \\
South Korea & 220 & 29 \\
Brazil & 146 & 22 \\
Israel & 128 & 28 \\
Belgium & 125 & 28 \\
Denmark & 121 & 26 \\
Taiwan & 110 & 19 \\
Sweden & 108 & 29 \\
Turkey & 82 & 17 \\
\hline
\end{tabular}

Table 2 The number of publications of the top 20 institutions

\begin{tabular}{lll}
\hline Institute & Country & Total articles \\
\hline University of London & England & 188 \\
University of Toronto & Canada & 182 \\
Imperial College London & England & 164 \\
Harvard University & USA & 150 \\
University of California System & USA & 146 \\
McGill University & Canada & 113 \\
University College London & England & 110 \\
Centre NationaL De Larecherche Sci- & France & 102 \\
entifique & & \\
Johns Hopkins University & USA & 95 \\
University of Copenhagen & Denmark & 85 \\
Pennsylvania Commonwealth System of & USA & 82 \\
Higher Education PCSHE & & \\
University of Southern California & USA & 79 \\
University of Washington & USA & 77 \\
University of Zurich & Switzerland & 76 \\
University of Washington Seattle & USA & 75 \\
University of Texas System & USA & 73 \\
Rigshospitalet & Denmark & 68 \\
Tel Aviv University & Israel & 65 \\
Ruprecht Karls University Heidelberg & Germany & 64 \\
University of Sydney & Australia & 64 \\
\hline
\end{tabular}

Average citations per item

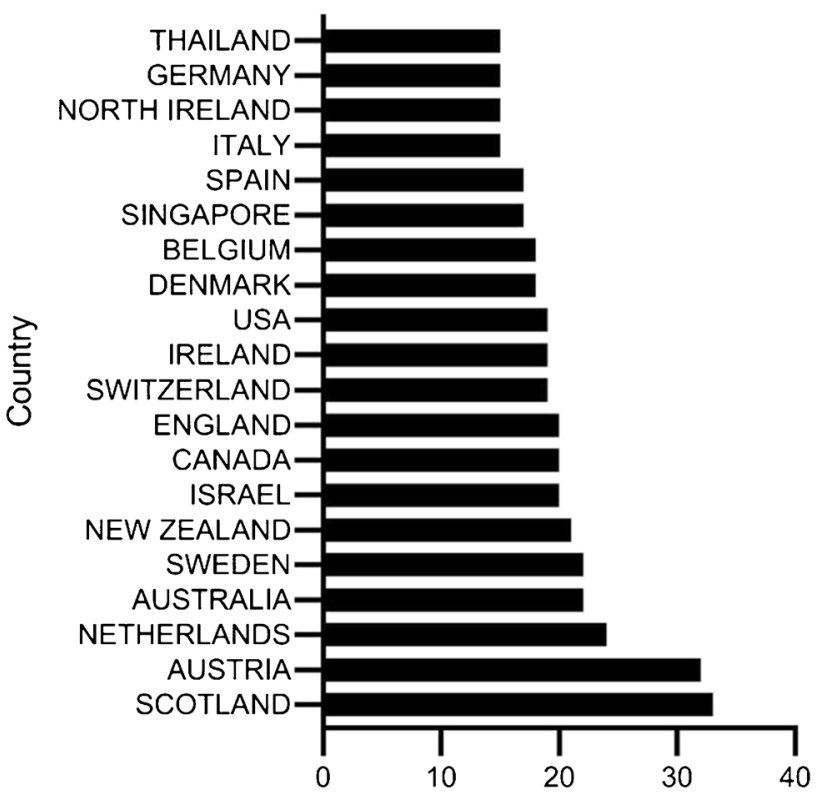

Fig. 11 The average numbers of citations per publication for the indicated countries

\section{H-Index Values}

Publications included in this study from the USA had the highest $\mathrm{H}$-index value (81), followed by those from England and Canada (55), Australia (41), the Netherlands (41), and Italy (40) (Fig. 13).

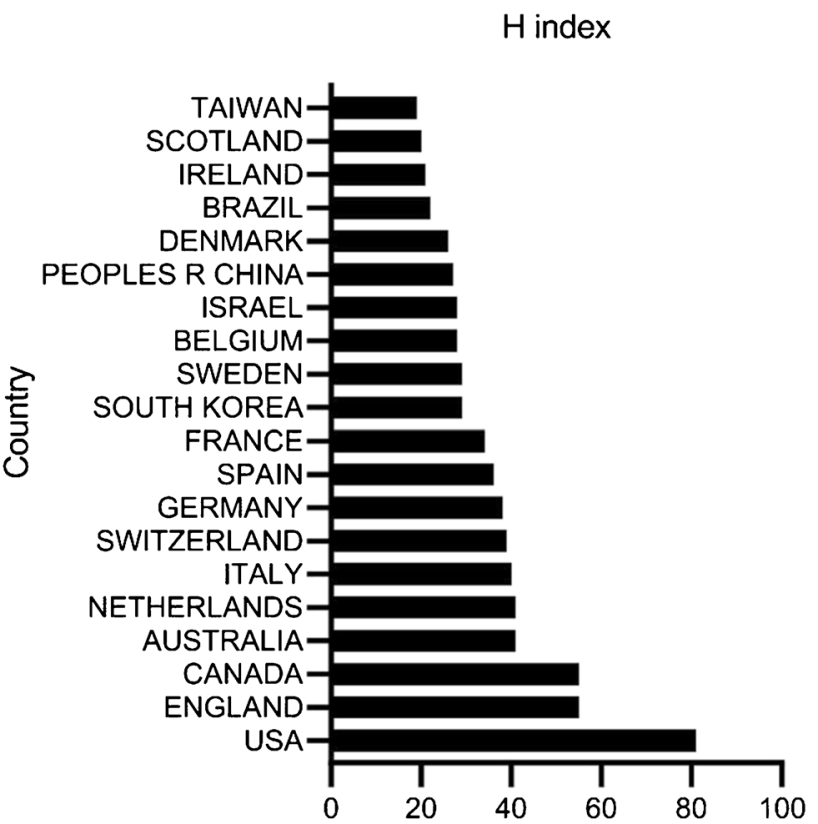

Fig. $12 \mathrm{H}$-index values for publications from the indicated countries. Bibliographic coupling analysis of global research trends pertaining to studies of the use of AR, VR, and MR technologies in surgery. 
neuroquantology

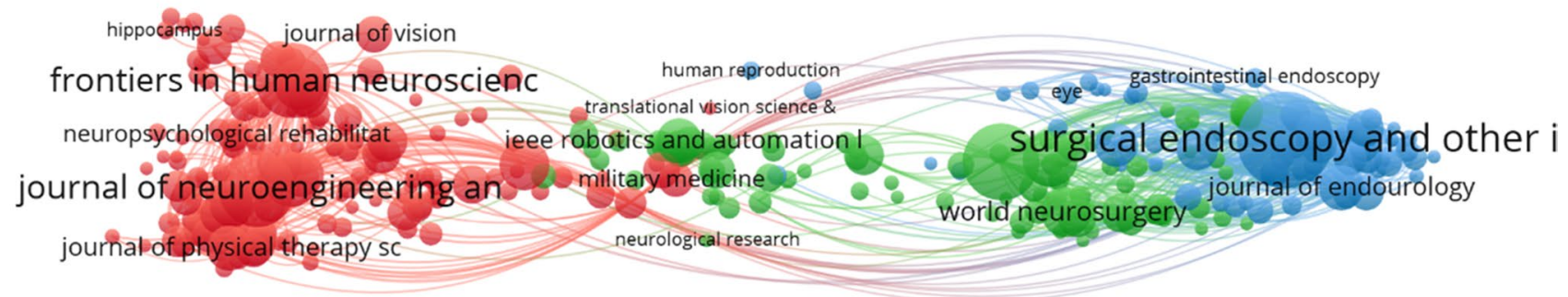

\& VOSviewer

Fig. 13 The mapping of 308 identified journals that have published studies in this research field

\section{Bibliographic Coupling Analysis}

\section{Journals}

Bibliographic coupling is a metric that can be used to detect similarity relationships between studies based upon their citations. When two studies both cite another study, this suggests that they share a related research focus. Bibliographic coupling analyses pertaining to journals, institutions, and countries were associated with relevant studies using VOS viewer, with individual spheres in the resultant mapping diagrams corresponding to the corresponding journals/

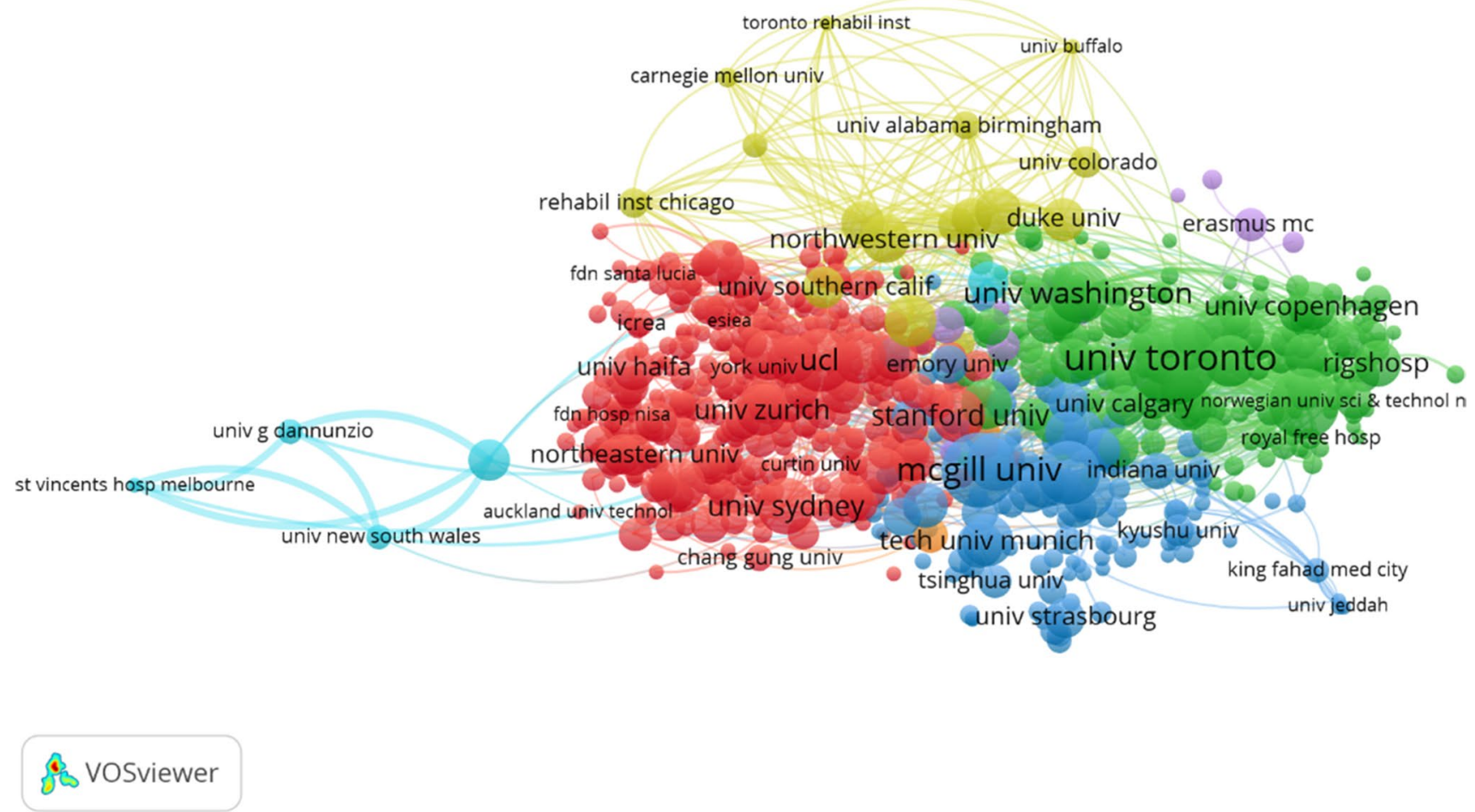

Fig. 14 The mapping of 64 countries associated with publications in this field. Lines between two points indicate a relationship between the two connected items, with thicker lines corresponding to a closer link between these journals, institutions, or countries. Co-authorship analysis of global studies pertaining to the use of AR, VR, and MR in surgery 


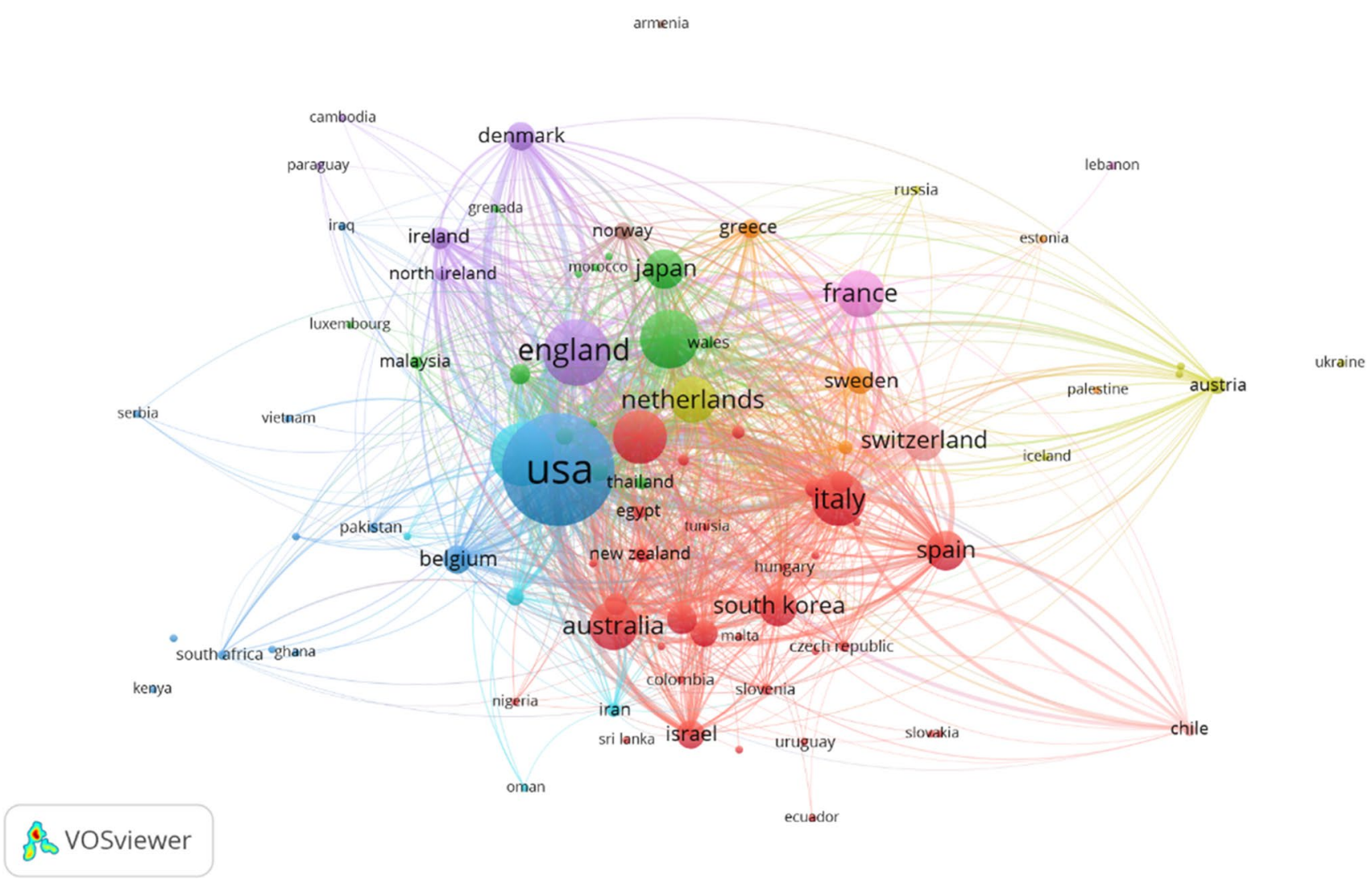

Fig. 15 The mapping of 676 institutions that have published research in this field

institutions/countries, and with sphere size corresponding to total linkage strength (TLS) between individual elements, with greater numbers of links being indicative of stronger relationships between pairs of elements [22]. This approach can also be employed to conduct co-authorship, co-citation, and co-occurrence analyses.

The TLS for 308 journals publishing relevant articles pertaining to the use of VR, AR, and MR in surgery are shown in Fig. 14. The journals with the greatest TLS values were Surgical Endoscopy And Other Interventional Techniques (Impact Factor, IF =3.149, 2019; TLS $=88,700)$, Journal Of Neuroengineering and Rehabilitation (IF $=3.519$, 2019; TLS $=48.242)$, Frontiers In Human Neuroscience $(\mathrm{IF}=2.673,2019 ; \mathrm{TLS}=30.797)$, American Journal of Surgery $(\mathrm{IF}=2.125,2019 ;$ TLS $=25,893)$, and Annals of Surgery $(\mathrm{IF}=10.13,2019$; $\mathrm{TLS}=25.739)$.

\section{Institutions}

Data from 676 institutions with 5 or more relevant publications were visualized using VOS viewer (Fig. 15), revealing the institutions with the greatest TLS values to be University of Toronto (TLS $=182,489$ ); Imperial College of
Science, Technology, and Medicine (TLS $=120,917$ ); McGill University (TLS $=100,914$ ); University of Washington (TLS $=83,718$ ); and Technische Universiteit Delft $(\mathrm{TLS}=60,754)$.

\section{Countries}

Papers from 64 countries were assessed with VOS viewer (Fig. 16), revealing the countries with the greatest TLS values to be the USA (TLS $=686,681$ ), England (TLS $=389,195)$, Canada (TLS $=343,385)$, Italy (TLS $=217,182)$, and Australia $(\mathrm{TLS}=201,596)$.

\section{Co-authorship Analyses}

Co-authorship analyses offer a means of assessing the relatedness of particular researchers or other items based upon numbers of co-authored publications, providing insights into collaboration among researchers, institutions, and countries in a given field of interest [21]. These analyses offer value as a means of providing researchers with 


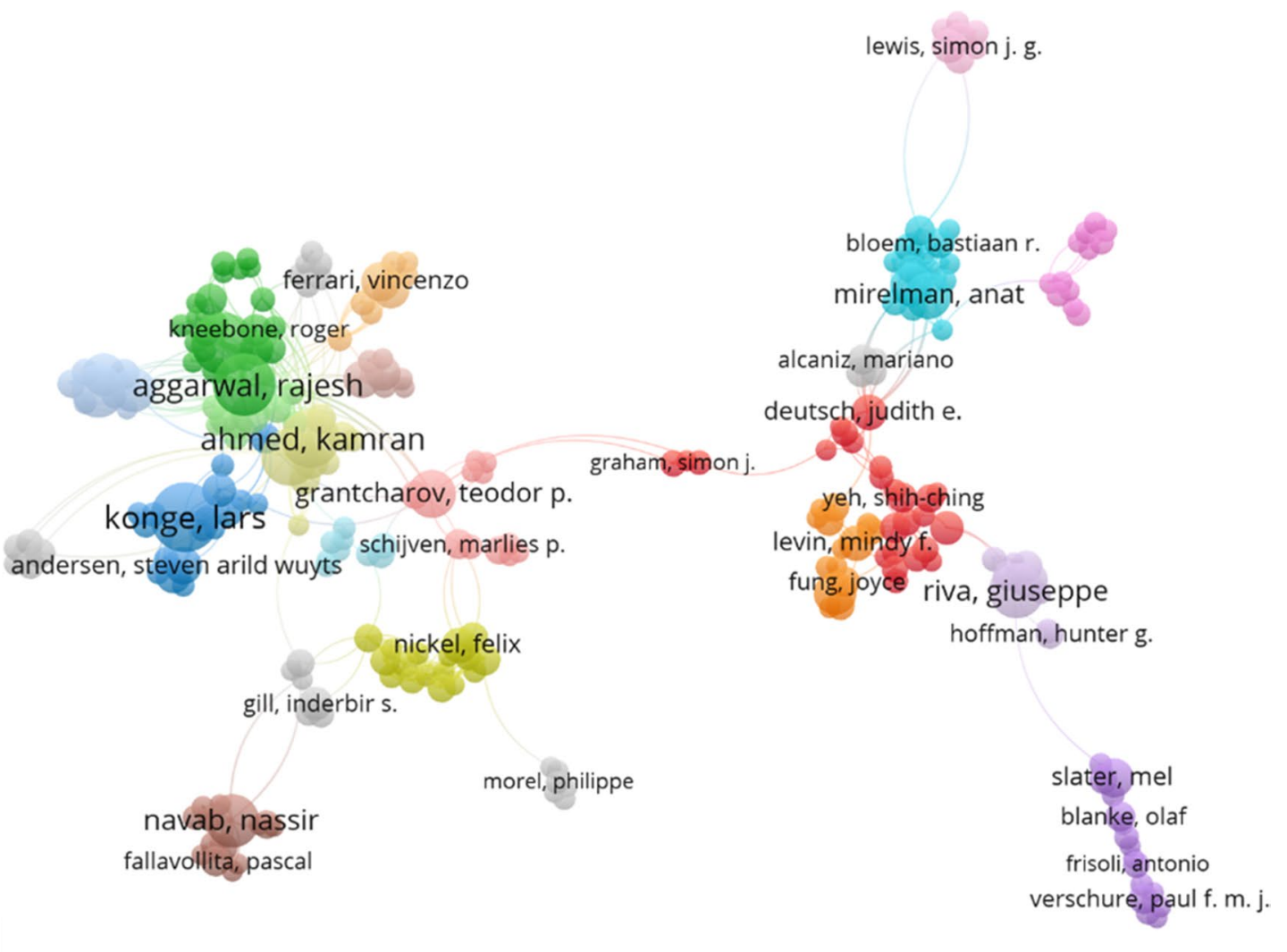

Fig. 16 Co-authorship mapping for 581 authors associated with studies in this field of research

valuable insights regarding potential collaborators, collaborative research group developments, and academic exchanges [22].

\section{Authors}

In total, we analyzed 581 different authors with a minimum of 5 publications using VOS viewer (Fig. 17). The authors with the greatest TLS were Calabro Rocco Salvatore (TLS = 133), De Luca Rosaria (TLS = 104), Ahmed Kamran (TLS $=94)$, Darzi Ara (TLS=93), and Bramanti Placido $(\mathrm{TLS}=89)$.

\section{Institutions}

In total, VOS viewer was used to analyze publications from 676 institutions (Fig. 18). The institutions with the greatest TLS values were the University of Toronto (TLS $=268$ ), McGill University (TLS $=180$ ), Harvard University (TLS = 138), Tel Aviv University (TLS = 129), and Johns Hopkins University (TLS $=128$ ).

\section{Countries}

In total, 99 different countries were analyzed with VOS viewer (Fig. 19). The countries with the greatest TLS values were the USA (TLS $=928)$, England $(T L S=645$ ), Italy (TLS $=446)$, Germany (TLS $=445)$, and Canada (TLS $=418)$.

\section{Co-citation Analysis}

Co-citation analyses are conducted by evaluating the number of times studies are cited together, thereby offering insights into correlations and similarities between articles and providing a firm knowledge base for a given field of interest.

\section{Authors}

In total, 922 authors with greater than 10 publications were next analyzed (Fig. 20), revealing the authors with the greatest TLS values to be Aggarwal R (TLS $=21.787)$, Gallagher AG $(\mathrm{TLS}=15.005)$, Hoffman HG $(\mathrm{TLS}=13.695)$, 


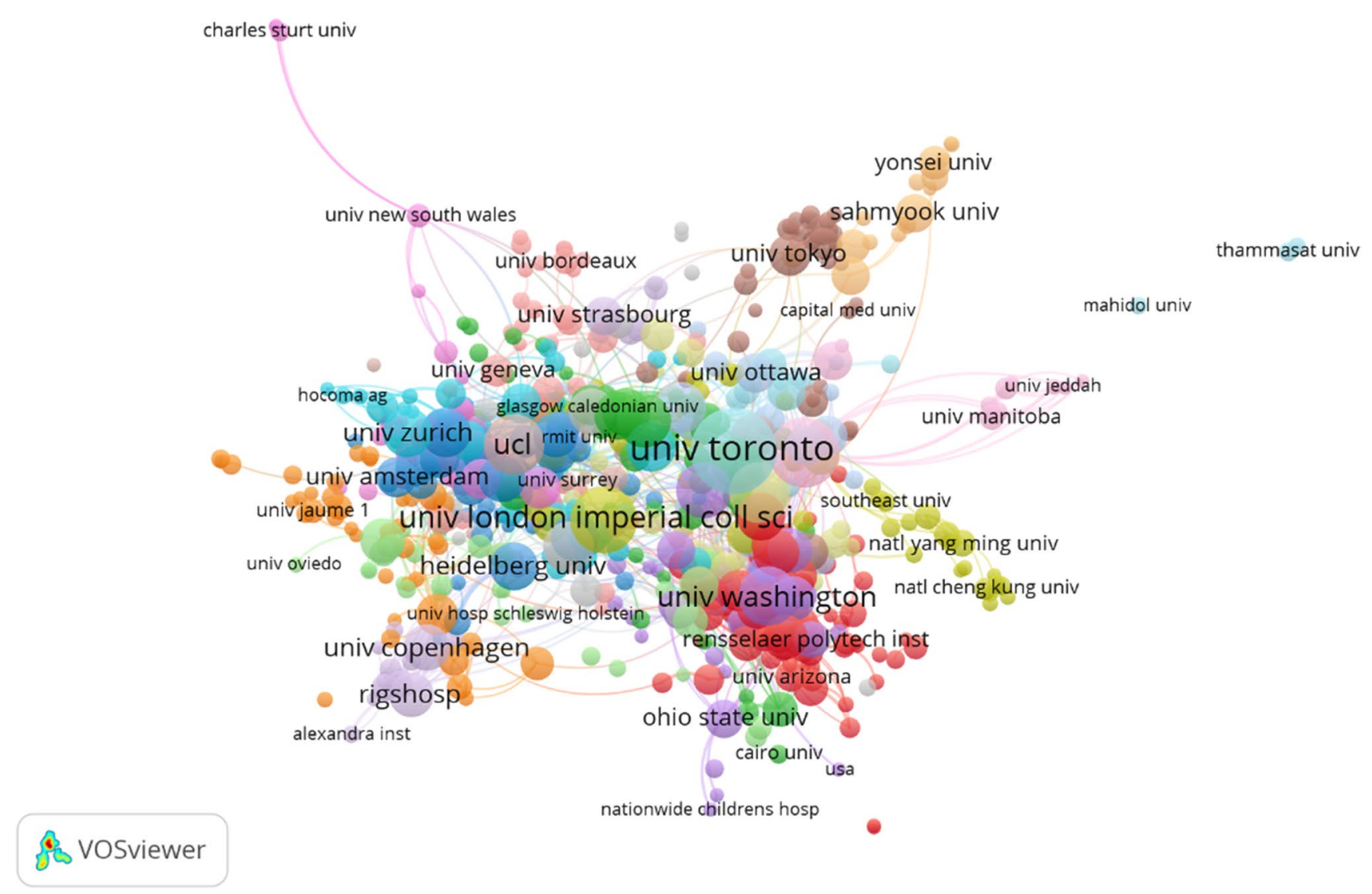

Fig. 17 Co-authorship mapping was conducted for 676 institutions with studies in this field of research

Grantcharov TP (TLS $=13.070)$, and Stefanidis D (TLS = 12.829).

\section{Journals}

Only journals with a least 20 citations were analyzed. The TLS values for these 1476 journals are shown in Fig. 21. Among these top journals, the largest TLS values are Surgical Endoscopy And Other Interventional Techniques (TLS = 202,529), Archives Of Physical Medicine And Rehabilitation (TLS =164,092), PLOS One (TLS =147,507), International Journal Of Stroke (TLS =131,524), and Neurorehabilitation And Neural Repair (TLS = 130,230).

\section{Co-occurrence Analysis}

Co-occurrence analyses offer a means of summarizing large numbers of research articles and predicting future trending topics in a given field, thereby elucidating and guiding scientific progress. To perform such a co-occurrence analysis, we used VOS viewer to visualize keywords used 10 or more times in the abstracts and titles of identified publications (Fig. 22). Overall, we identified 952 keywords that were clustered into five rough clusters pertaining to Neurological Research, Surgical Techniques, Technological Products, Rehabilitative Medicine, and Clinical Therapy, suggesting these to be the most prominent topics in this research field to date. The most frequently utilized keywords in the "Neurological Research" cluster included virtual reality, Alzheimer's disease, Parkinson's disease, brain, and movement. In the "Surgical Technique" cluster, the most commonly utilized keywords were performance, validation, simulation, surgery, and education, while in the "Technological Products" cluster, they were augmented reality, system, visualization, accuracy, and guidance. Similarly, the primary keywords for the "Rehabilitative Medicine" cluster were virtual reality, rehabilitation, balance, stroke, and exercise, while for the "Clinical Therapy" cluster, they were children, therapy, video games, distraction, and interventions.

VOS viewer was then used to color code these keywords based upon the frequency with which they appeared in the studies included in this analysis (Fig. 23), with blue indicating that keyword appeared at earlier time points and red indicating a later appearance. Prior to 2005, more studies focused on keywords including "Virtual Reality," 


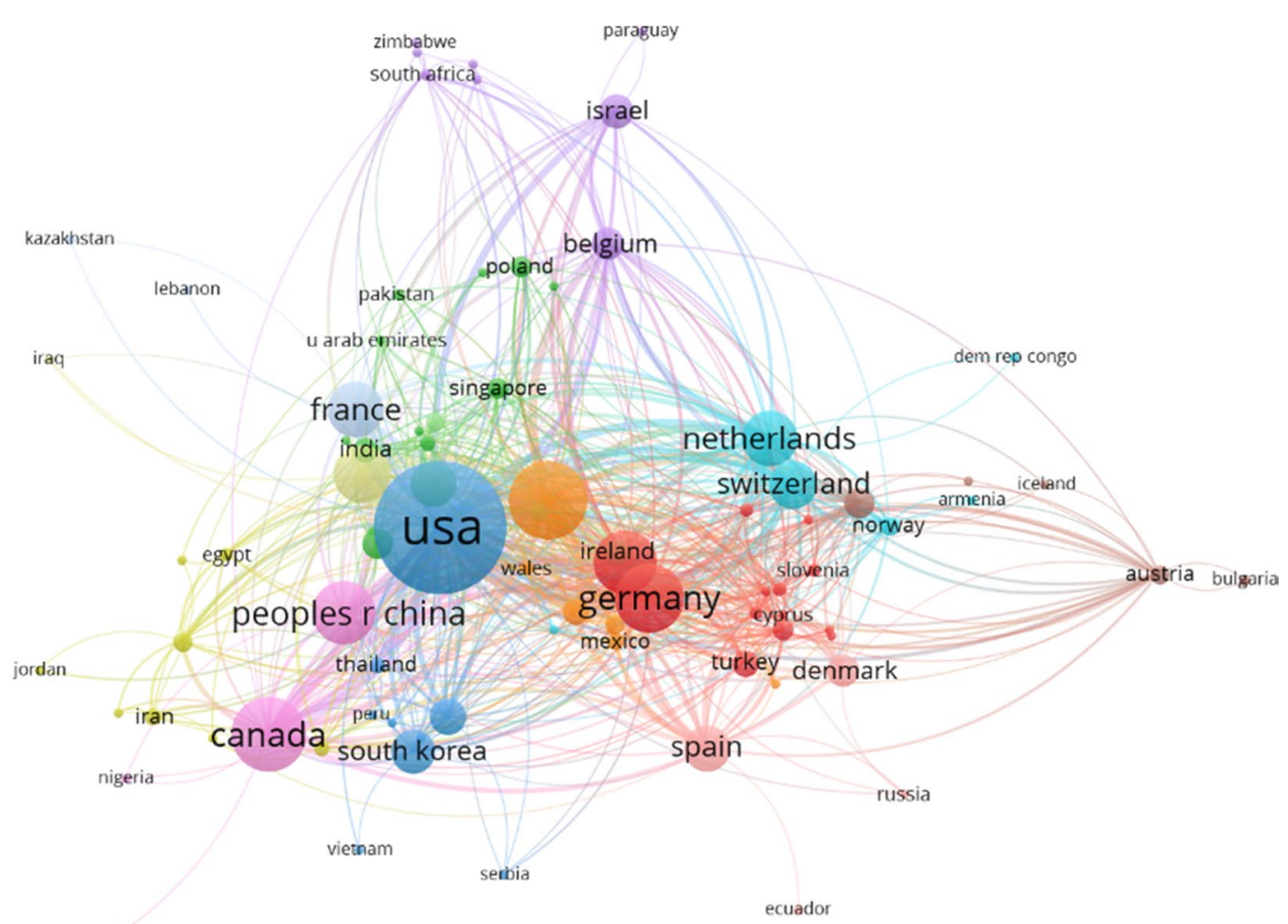

\& VOSviewer

Fig. 18 Co-authorship mapping was conducted for 99 countries with studies in this field of research. Point sizes correspond to co-authorship frequencies, with lines between points indicating an established collaboration between authors, institutions, or countries. Thicker lines correspond to closer collaborations between any two given authors, institutions, or countries. Co-citation analysis of global research trends pertaining to the use of AR, VR, and MR in surgery

space over the past decade, and our analysis suggests that this is an expanding area of interest that will be associated with the publication of rising numbers of high-quality articles in the coming years.

\section{Global Publication Quality and Status}

A global analysis of publications in this field revealed that the USA is responsible for the highest overall number of published papers, with the US Department of Health and Human Services being one of the most prominent funding sources in this research space. Perhaps unsurprisingly, surgical medicine was the most heavily researched branch of this field, and the USA exhibited the greatest contribution to this field with respect to total citations, H-index values, and publication numbers. England additionally exhibited the highest total citation counts of any countries contributing to this field, highlighting the USA and England as global leaders in the use of VR, AR, and MR in surgical medicine. Canada ranked third in terms of publication volume, $\mathrm{H}$-index, 99 different countries have published studies in this research 
verwoerd-dikkeboom, cm

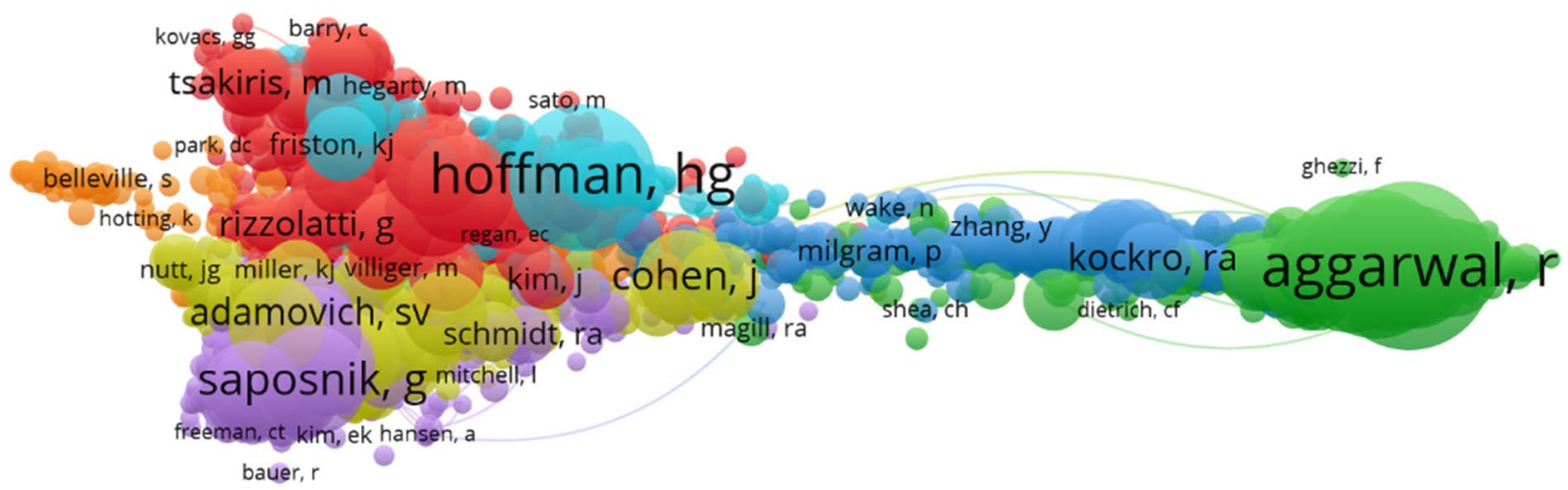

sanders, da

\section{VOSviewer}

Fig. 19 An author co-citation analysis was performed for studies in this research space, with points of a given color corresponding to shared research directions

and total citations in this field, while China was the sixth most published country but was only 16 th with respect to the average number of citations per publication. This may be attributable to the historical emphasis on quantity rather than quality in the Chinese academic sector. However, the continued expansion of Chinese government-led medical research funding is likely to improve average study quality, helping China establish itself as a global research leader in this field.

When we conducted bibliographic coupling analyses of journals, institutions, and countries in this research space, we identified Surgical Endoscopy And Other Interventional Techniques, International Orthopaedics, International Journal Of Computer Assisted Radiology And Surgery, and Frontiers In Human Neuroscience as popular journals for the publication of studies evaluating the use of VR, AR, and MR in surgical contexts. Monitoring publications in these journals may thus be an effective means of tracking research trends in this field. We additionally found that the University of London was a leader in this field of research, while Imperial College London ranked third overall, consistent with the strong contributions of England to this research area. Harvard University and the University of California System were the top research institutions in the USA, while the University of Toronto in Canada ranked second in an institutional coupling analysis. These findings emphasize the close relationship between top-ranked universities and the overall academic level of a given country. We found that Aggarwal R, Konge $\mathrm{L}$, and Darzi A were the researchers who have made the greatest contributions to this field, suggesting that monitoring their future publications will highlight key trends for future development in this space.

These analyses suggest that the University of Toronto, McGill University, and Harvard University represent optimal collaborative partners and co-authors for individuals conducting research on the use of VR, AR, and MR in surgery. Surgical Endoscopy And Other Interventional Techniques, Archives Of Physical Medicine And Rehabilitation, and PLOS One were the three most frequently cited journals in the field, while Aggarwal R, Gallagher AG, and Hoffman HG were the most highly cited and widely recognized researchers conducting these studies.

\section{VR, AR, and MR Surgical Research Focus Areas}

A co-occurrence network diagram generated herein highlighted five key research trends in this field: neurological research, surgical techniques, technological products, rehabilitative medicine, and clinical therapy.

At a more focused level, MR technologies have been deployed in the context of surgical education, enabling surgeons to more rapidly expand their skillsets to ensure career growth 


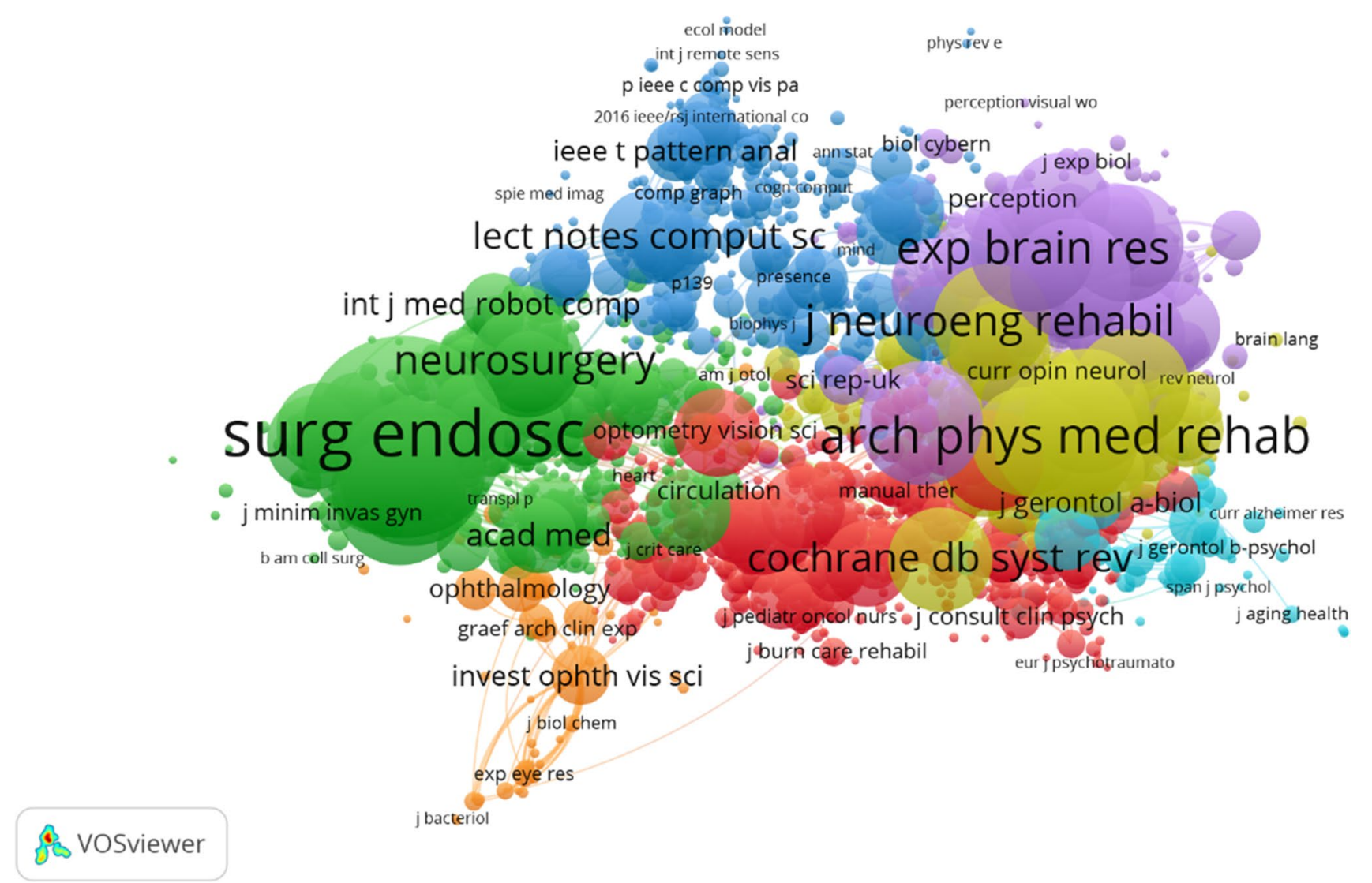

Fig. 20 Co-cited journals in this research space were mapped, with point size corresponding to citation frequency. A line between two points indicates that both were cited in a given paper or journal,

[24]. New AR, VR, and MR tools are also used with increasing frequency to ensure that patients and their families understand pertinent surgical procedures, reducing the potential for doctorpatient miscommunication $[25,26]$. Preoperative imaging technologies offer a clear view of the surgical site and surrounding tissues, enabling safer and more precise surgical planning [27, 28], and VR, AR, and MR tools are therefore being used with increasing frequency in clinical surgery in disciplines such as neurosurgery, orthopedics, plastic surgery, otorhinolaryngology, thoracic surgery, and urology $[29,30]$. These approaches can reduce operative durations, radiation doses, and incision sizes, thereby providing patients with increasingly high-quality care. VR-based clinical rehabilitation platforms have also been built and applied in the field of orthopedics [31], while ARbased developments have improved teleconsultation quality and medical resource sharing [32].

At a high level, approaches to combining AR and 3D printing technologies appear to be a growing area of interest in surgical medicine [33], particularly in neurosurgery and orthopedics. At the same time, image-based surgical navigation strategies are being increasingly employed in the contexts of vascular surgery, brain pacemakers, and epilepsy electrode with shorter lines indicating a closer link between two given papers or journals. Co-occurrence analysis of global trends pertaining to the use of AR, VR, and MR technologies in the field of surgical research

implantation [34]. AR-based head-mounted display (HMD) units leveraging holographic imaging technology are also being employed in top operating rooms throughout the world [35]. Growing trends suggest that the development of ARbased semi- or fully automated robots with integrated scanners and surgical arms is likely to dramatically influence the surgical field in the coming years [36]. Novel extended reality (XR) technologies incorporating elements of VR, AR, or MR are forecast to be used with rising frequency by surgeons as these tools undergo further development $[3,37]$, and the integration of cloud-based services and healthcare data will ensure that these XR platforms are fully optimized to facilitate high-quality patient care. The results of this bibliometric analysis emphasize the crucial need for high-quality studies in this research space, highlighting key areas for additional experimental or clinical research and funding infusion as appropriate.

Colors in the generated density visualization map correspond to the frequencies of occurrence for particular keywords. This analysis suggested that Surgical Techniques and Technological Products are likely to be popular research subjects in this field, with many studies pertaining to extended 


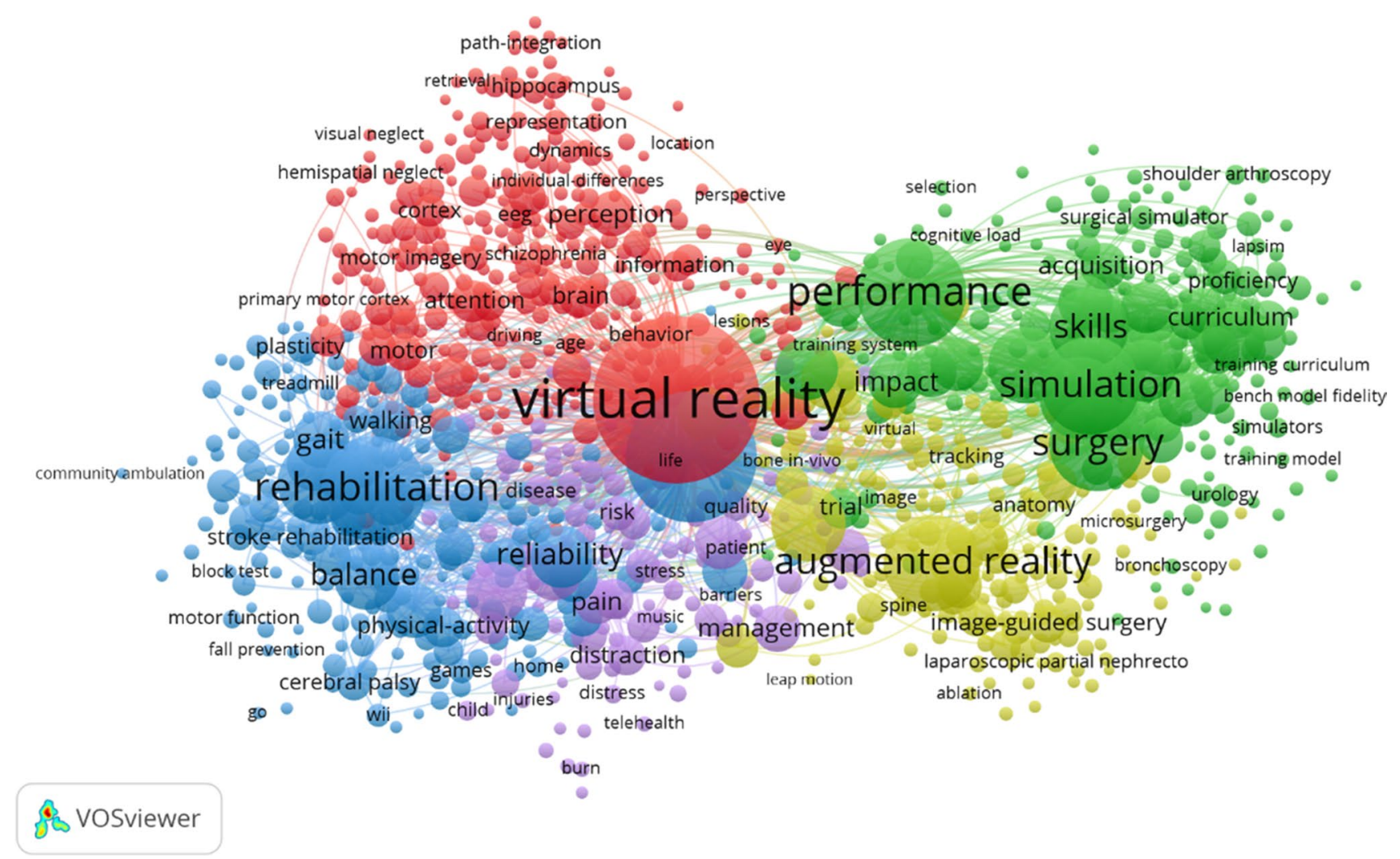

Fig. 21 Keywords relevant to this analysis were mapped, with the size of individual points corresponding to frequency values. Overall, ;these keywords clustered into five categories: Neurologi-

reality, navigation, and holographic images having emerged in recent years.

\section{Strengths and Limitations of This Study}

By employing bibliometric and visualization techniques to highlight trends in the use of AR, VR, and MR technologies in the field of surgical research, we have herein provided a robust overview of this clinically important field. It is worth mentioning that, through the processing of GraphPad Prism and VOSviewer, the content and results of the above research can be presented to readers more intuitively and vividly.

Even so, there are certain limitations to our analyses. For one, we excluded studies not published in English, potentially introducing a language bias into our findings. In addition, we only analyzed studies published as of 13 October 2020, and our analyses thus fail to reflect more recent studies in this field. Future analyses should therefore be extended to the non-English literature to better understand global trends in this research field. At the same time, the literature we selected was all articles or reviews, and did not include conferences, books, and other forms, which would make our cal Research (red; upper left), Surgical Techniques (green; upper right), Technological Products (yellow; lower right), rehabilitative medicine (blue, lower left), and clinical therapy (purple, lower left)

research results lack certain comprehensiveness. Similarly, when we searched, we selected the Web of Science database and did not search other databases, which would also lead to certain deviation in the inclusion of literature. We should avoid the above problems in the future research.

\section{Bias Analysis}

In the selection of research content, this paper did not include other research results other than treatises and reviews, and only English articles were selected instead of articles in other languages, and only the WOS database was referred to in the selection of database, so it was suspected of selection bias. Because the data extraction and statistics of the paper were completed by the two authors, the inherent investigator bias was suspected.

\section{Conclusions}

Overall, our study provides high-level overview of current trends in the application of AR, VR, and MR technologies in surgical research. While the USA remains the 


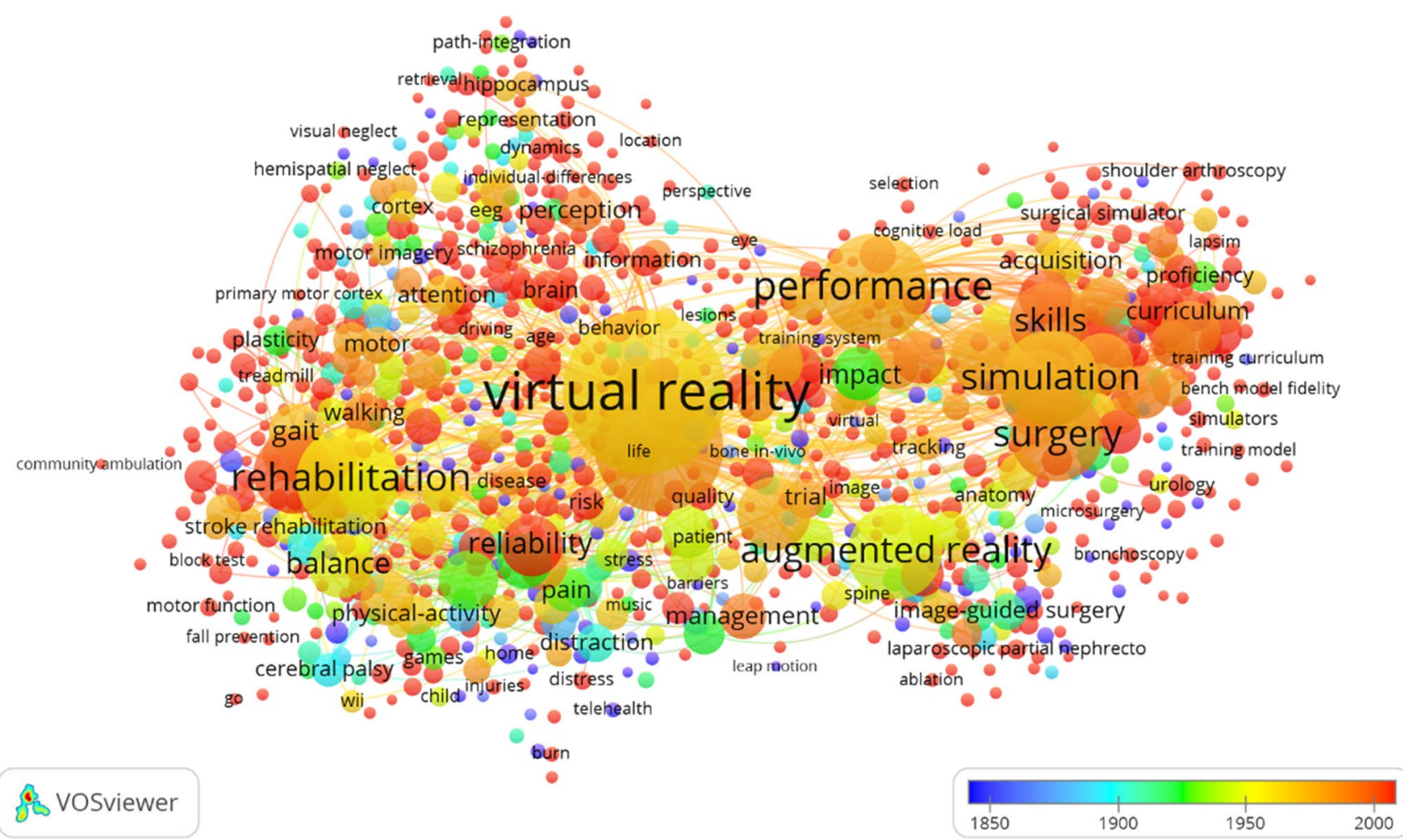

Fig. 22 Keyword distributions based upon mean appearance frequency, with keywords in blue appearing earlier than keywords in red or yellow

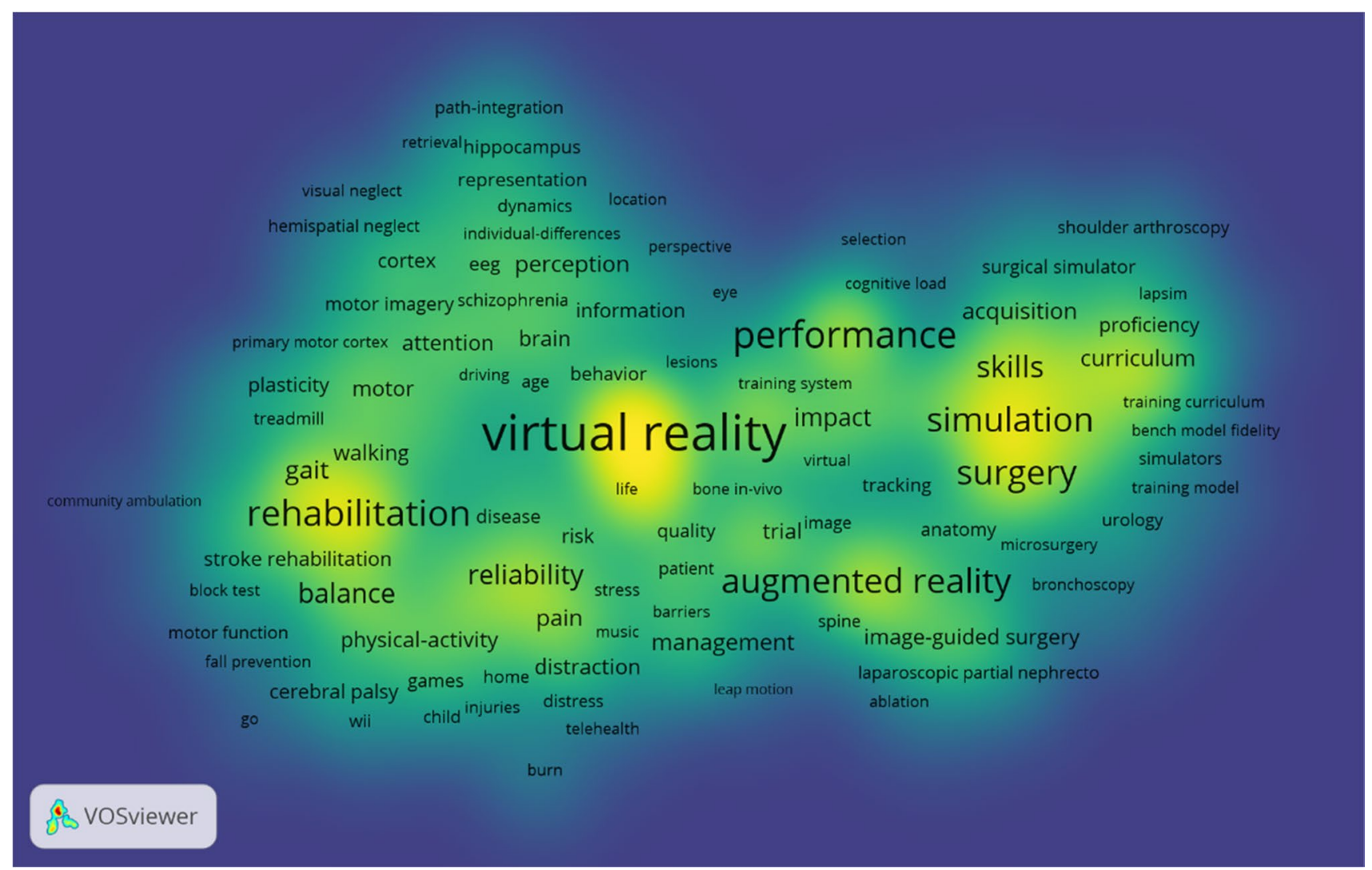

Fig. 23 A density visualization map was generated based upon the times a given keyword occurred, which defined the area for a given term, with warmer colors corresponding to greater numbers of occurrences 
clear leader in this field, followed by England, Australia is actively growing as a major in this field that prioritizes academic quality and is thereby actively influencing associated scientific norms. Key areas of future research are forecast to include extended reality, navigation, and holographic image-related technologies. Through such analyses and the predicted overall expansion in the number of studies in this field, we predict that these technologies will continue to benefit medicine and to thereby improve the available quality of care for all patients.

Author Contribution Xin Lv and Bin Wang conceived the research; Jing Zhang and $\mathrm{Na} \mathrm{Yu}$ collected the data and prepared the manuscript; Jing Zhang and $\mathrm{Na} \mathrm{Yu}$ analyzed the data; $\mathrm{Na}$ Yu provided techniques and advice.

Funding This study was supported by a grant from National Nature Science Foundation of China (No. 81802204).

Data Sharing No additional data are available.

\section{Declarations}

Ethics Approval Ethical approval of the study was not necessary.

Competing Interests The authors declare no competing interests.

Patient and Public Involvement No patient involved. This review was not registered. The protocol was not prepared.

\section{References}

1. Mertz L (2019) Virtual reality pioneer Tom Furness on the past, present, and future of VR in health care. IEEE Pulse 10(3):911. https://doi.org/10.1109/MPULS.2019.2911808

2. Sutherland J et al (2019) Applying modern virtual and augmented reality technologies to medical images and models. J Digit Imaging 32(1):38-53. https://doi.org/10.1007/s10278-018-0122-7

3. Sakai D et al (2020) Augmented, virtual and mixed reality in spinal surgery: a real-world experience. J Orthop Surg. https:// doi.org/10.1177/2309499020952698

4. Saito $\mathrm{Y}$ et al (2020) Intraoperative 3D hologram support with mixed reality techniques in liver surgery. Ann Surg 271(1):e4 e7. https://doi.org/10.1097/SLA.0000000000003552

5. Yoshida S et al (2019) Mixed reality computed tomography-based surgical planning for partial nephrectomy using a head-mounted holographic computer. Int J Urol 26(6):681-682. https://doi.org/10.1111/iju.13954

6. Verhey JT, et al. (2020) Virtual, augmented, and mixed reality applications in orthopedic surgery. Int $\mathrm{J}$ Med Robot 16(2):e2067. https://doi.org/10.1002/rcs.2067

7. $\mathrm{Hu} \mathrm{HZ}$ et al (2019) Application and prospect of mixed reality technology in medical field. Curr Med Sci 39(1):1-6. https://doi. org/10.1007/s11596-019-1992-8

8. Mao X, et al. (2020) The status and trends of coronavirus research: a global bibliometric and visualized analysis. Medicine 99(22):e20137. https://doi.org/10.1097/MD.0000000000020137

9. Tijssen RJ, Winnink J (2016) Twenty-first century macro-trends in the institutional fabric of science: bibliometric monitoring and analysis. Scientometrics 109(3):2181-2194. https://doi.org/10. 1007/s11192-016-2041-z

10. Ekinci $\mathrm{S}$ et al (2015) Letter to the Editor regarding analysis of changing paradigms of management in 179 patients with spinal tuberculosis during a 12-year period and proposal of a new management algorithm. World Neurosurg 84(6):2072. https://doi.org/ 10.1016/j.wneu.2014.12.003

11. Pu QH, Lyu QL, Su HY (2016) Bibliometric analysis of scientific publications in transplantation journals from Mainland China, Japan, South Korea and Taiwan between 2006 and 2015. BMJ Open 6(8): p. e011623. https://doi.org/10.1136/bmjop en-2016-011623

12. Chien TW, et al. (2019) Choropleth map legend design for visualizing the most influential areas in article citation disparities: a bibliometric study. Medicine (Baltimore) 98(41): p. e17527. https:// doi.org/10.1097/MD.0000000000017527

13. Zyoud SH (2016) Global research trends of Middle East respiratory syndrome coronavirus: a bibliometric analysis. BMC Infect Dis 16:255. https://doi.org/10.1186/s12879-016-1600-5

14. Wang B et al (2019) The state of exosomes research: a global visualized analysis. Biomed Res Int 2019:1495130. https://doi.org/10. $1155 / 2019$

15. Cislo-Pakuluk A, Marycz K (2017) A promising tool in retina regeneration: current perspectives and challenges when using mesenchymal progenitor stem cells in veterinary and human ophthalmological applications. Stem Cell Rev Rep 13(5):598602. https://doi.org/10.1007/s12015-017-9750-4

16. Zhai X, et al. (2017) Global research trends in spinal ultrasound: a systematic bibliometric analysis. BMJ Open 7(10):e015317. https://doi.org/10.1136/bmjopen-2016-01531717

17. Aggarwal A et al (2016) The state of lung cancer research: a global analysis. J Thorac Oncol 11(7):1040-1050. https://doi. org/10.1016/j.jtho.2016.03.010

18. Pei W et al (2019) Research trends of acupuncture therapy on insomnia in two decades (from 1999 to 2018): a bibliometric analysis. BMC Complement Altern Med 19(1):225. https://doi. org/10.1186/s12906-019-2606-519

19. Zou X, Yue WL, Vu HL (2018) Visualization and analysis of mapping knowledge domain of road safety studies. Accid Anal Prev 118:131-145. https://doi.org/10.1016/j.aap.2018.06.010

20. Bertoli-Barsotti L, Lando T (2017) A theoretical model of the relationship between the $\mathrm{h}$-index and other simple citation indicators. Scientometrics 111(3):1415-1448. https://doi.org/10.1007/ s11192-017-2351-9

21. Mao X, et al. (2020) A global bibliometric and visualized analysis in the status and trends of subchondral bone research. Medicine (Baltimore) 99(22):e20406. https://doi.org/10.1097/MD.00000 00000020406

22. Mao XJ, et al. (2020) The status and trends of coronavirus research: a global bibliometric and visualized analysis. Medicine. https://doi.org/10.1097/MD.0000000000020137

23. Zhai X, Wang Q, Li M (2016) Tu Youyou's Nobel Prize and the academic evaluation system in China. The Lancet 387(10029). https://doi.org/10.1016/S0140-6736(16)30261-6

24. Stefan P et al (2018) Team training and assessment in mixed reality-based simulated operating room: current state of research in the field of simulation in spine surgery exemplified by the ATMEOS project. Unfallchirurg 121(4):271-277. https://doi.org/ 10.1007/s00113-018-0467-x

25. Wu X et al (2018) Mixed reality technology launches in orthopedic surgery for comprehensive preoperative management of complicated cervical fractures. Surg Innov 25(4):421-422. https://doi. org/10.1177/1553350618761758

26. Lu K et al (2018) Use of short message service and smartphone applications in the management of surgical patients: a systematic review. 
Telemed J E Health 24(6):406-414. https://doi.org/10.1089/tmj.2017. 0123

27. Wu X et al (2018) Mixed reality technology-assisted orthopedics surgery navigation. Surg Innov 25(3):304-305. https://doi. org/10.1177/1553350618771413

28. Sauer IM et al (2017) Mixed reality in visceral surgery: development of a suitable workflow and evaluation of intraoperative use-cases. Ann Surg 266(5):706-712. https://doi.org/10.1097/ SLA.0000000000002448

29. Incekara $F$ et al (2018) Clinical feasibility of a wearable mixedreality device in neurosurgery. World Neurosurg 118:e422e427. https://doi.org/10.1016/j.wneu.2018.06.208

30. Lee SC et al (2017) Multi-modal imaging, model-based tracking, and mixed reality visualisation for orthopaedic surgery. Healthc Technol Lett 4(5):168-173. https://doi.org/10.1049/htl. 2017.0066

31. Cabana $\mathrm{F}$ et al (2016) Is an in-home telerehabilitation program for people with proximal humerus fracture as effective as a conventional face-to face rehabilitation program? A study protocol for a noninferiority randomized clinical trial. BMC Sports Sci Med Rehabil 8(1):27. https://doi.org/10.1186/s13102-016-0051-z

32. Wang S, et al. (2017) Augmented reality as a telemedicine platform for remote procedural training. Sensors. https://doi.org/10. $3390 / \mathrm{s} 17102294$
33. Negrillo-Cárdenas J, Jiménez-Pérez J-R, Feito FR (2020) The role of virtual and augmented reality in orthopedic trauma surgery: from diagnosis to rehabilitation. Computer Methods and Programs in Biomedicine. https://doi.org/10.1016/j.cmpb.2020.105407

34. Drouin $\mathrm{S}$ et al (2016) IBIS: an OR ready open-source platform for image-guided neurosurgery. Int J Comput Assist Radiol Surg 12(3):363-378. https://doi.org/10.1007/s11548-016-1478-0

35. Sayadi LR et al (2019) The new frontier: a review of augmented reality and virtual reality in plastic surgery. Aesthet Surg J 39(9):1007-1016. https://doi.org/10.1093/asj/sjz04336

36. Gorbanev I et al (2018) A systematic review of serious games in medical education: quality of evidence and pedagogical strategy. Med Educ Online 23(1):1438718. https://doi.org/10.1080/10872981.2018.14387 18

37. Southworth MK, Silva JR, Silva JNA (2020) Use of extended realities in cardiology. Trends Cardiovasc Med 30(3):143148. https://doi.org/10.1016/j.tcm.2019.04.005

Publisher's Note Springer Nature remains neutral with regard to jurisdictional claims in published maps and institutional affiliations. 